\title{
Business Cycles in European Post-Communist Countries
}

\author{
Karol Szomolányi ${ }^{1}$, Martin Lukáčik' ${ }^{1}$ Adriana Lukáčiková ${ }^{1}$
}

ABSTRACT The European post-communist countries that have integrated into the European Union are emerg-
ing market economies. However, their short-run economic performance does not correspond to
the observed business cycles of other global emerging markets. The business cycles of the studied
countries are longer, and their recessions are more pronounced. Moreover, economic activity in the
studied countries is relatively low and volatile, and the trade balance and government purchases have
a relatively significant countercyclical character. These are the core conclusions from our business
cycle study of the chosen European post-communist countries. In the study, we use both traditional
and contemporary business cycle definitions. Using traditional definitions, we determine the peaks,
troughs, expansions and contractions and quantify the durations and amplitudes of their expansions,
recessions and business cycles. Using contemporary definitions, we measure cyclical properties by
computing the first and second moments of the chosen macroeconomic cyclical components and
growth rates. Differences in the business cycles and characteristics between European post-com-
munist countries and other emerging countries are discussed with reference to the works of other
authors.

KEY WORDS: business cycles, emerging markets, European post-communist countries

JEL Classification: E32, O52

${ }^{1}$ University of Economics in Bratislava - Department of Operations Research and Econometrics, Slovakia

\section{Introduction}

In this paper, we analyze the cyclical properties of selected post-communist countries in the European Union using two simple characteristics. First, using the definition of Burns and Mitchell (1946), we identify business cycles based on turning points in the aggregate time series; second, using Lucas's (1977) definition, we identify business cycles based on movements about trends. The definition of Burns and Mitchell (1946) is

Correspondence concerning this article should be addressed to: Karol Szomolányi, University of Economics in Bratislava - Department of Operations Research and Econometrics, Dolnozemská cesta 1, Bratislava 852 35, Slovakia. E-mail: karol.szomolanyi@euba.sk used to identify business cycles, whereas the definition of Lucas (1977) is used to discern the cyclical properties of the chosen aggregate time series by computing the moments of cyclical components. In accordance with Lucas's definition, we state that the aggregate time series is pro-cyclical (counter-cyclical) if the cyclical component of the series is positively (negatively) correlated with the cyclical component of the output time series. If the cyclical component of the aggregate data series is not correlated with that of the output, the series is acyclical. The volatility of the data series is measured using the standard deviation of its cyclical component. The persistency of the data series is measured by the serial correlation coefficient of its cyclical component. 
The results obtained are compared with the results of analyses of business cycles around the world. Uribe and Schmitt-Grohé (2017) and Montiel (2013) state that the aforementioned simple business cycle characteristics differ across countries of different sizes, wealth, and openness. In the comparative analysis, we must consider that according to the definition of Uribe and Schmitt-Grohé (2017), all the studied postcommunist countries are emerging. Their average purchasing power parity (PPP) converted into GDP per capita over the period 1990-2009 falls within the $3,000-25,000$ range. Presented below is a summary of the Uribe and Schmitt-Grohé (2017) conception of the relationship between a country's wealth and its business cycle:

- business cycles in rich countries are approximately one-half as volatile as business cycles in emerging or poor countries.

- consumption volatility is higher in poor and emerging countries than in rich countries.

- the trade balance-to-output ratio is more negatively correlated with output as the level of economic development increases.

- the share of government consumption in relation to output is counter-cyclical in rich countries but acyclical in emerging and poor countries.

- expansions are on average longer in developed countries than in emerging countries.

- contractions are more pronounced in emerging countries than in developed countries.

The authors obtained the last two items in the above list from Calderón and Fuentes (2010). Uribe and Schmitt-Grohé (2017) also take into account the higher volatility of economic activity in small open countries than that in large countries.

Assuming that these simple characteristics of business cycles in emerging countries around the world coincide with those of post-communist countries, it is useful to review the empirical and theoretical literature analyzing the business cycles of emerging countries. Numerous authors have successfully explained many of the aforementioned characteristics. The basic principles can be understood based on a simple real business cycle model developed by Kydland and Prescott (1982), which is resistant to the Lucas (1976) critique. Mendoza (1991) made an important modification to the model by forming a real business cycle model of a small open economy to study Canadian business cycles. In interpreting the model, Uribe and SchmittGrohé identify certain theoretical principles to explain consumption volatility and trade balance cyclicality:

- the more persistent the productivity shocks are, the more volatile consumption is and thus the more counter-cyclical the trade balance is.

- the more pronounced capital costs are, the more pro-cyclical the trade balance is.

Using these principles, the authors tried to empirically explain the characteristics of business cycles in emerging countries. According to Aguiar and Gopinath (2007), high consumption volatility in emerging economies derives from non-stationary productivity shocks. However, García-Cicco, Pancrazi and Uribe (2010) argue that the data range used in the paper of Aguiar and Gopinath is too small to study the non-stationarity of the variables. Furthermore, using the persistent productivity shock principle, the real business cycle model of the small open economy with nonstationary output shocks predicts not only highly volatile consumption but also a relatively strongly counter-cyclical trade balance. The trade balance is indisputably less counter-cyclical in emerging countries than it is in developed countries.

García-Cicco et al. (2010) consider several shocks to explain the business cycles of emerging economies. In addition to various productivity shocks, agents in emerging and poor countries face interest rate and interest rate spread premium changes. According to the authors, in poor and emerging countries, interest rate and interest rate spread shocks are significant whereas non-stationary productivity shocks are negligible. Uribe and Yue (2006) show that interest rate and interest rate spread shocks significantly influence business cycles in poor and emerging countries. The interest rate spread is considered partially exogenous and partially endogenous. Access of domestic agents to international financial markets is better (and capital adjustment costs are lower) if domestic economic policy is credible. Agents may borrow funds at a relatively low interest rate, and the country's interest rate spread is low. Therefore, the reason for the high economic volatility of poor and emerging countries is international financial market friction. The low economic activity 

Table 1. Durations and Amplitudes of Contractions and Expansions in Three Country Groups

\begin{tabular}{|c|c|c|c|c|}
\hline & country groups & developed & emerging & post-communist \\
\hline \multirow{2}{*}{ durations } & contraction & 4.2 & 4.8 & 5.0 \\
\hline & expansion & 14.3 & 16.3 & 25.6 \\
\hline \multirow{2}{*}{ amplitudes } & contraction & 3.4 & 4.4 & 8.4 \\
\hline & expansion & 7.5 & 19.4 & 30.4 \\
\hline \multirow{2}{*}{ cumulative gain/loss } & contraction & 9.2 & 12.8 & 33.0 \\
\hline & expansion & 55.1 & 224.3 & 445.3 \\
\hline
\end{tabular}

Note: The quarterly time series of post-communist countries for the period 1995-2013 are adapted from "GDP and main components (output, expenditure and income)" by Eurostat database (2014). Retrieved from http://appsso.eurostat.ec.europa.eu/ nui/show.do?dataset=namq_10_gdp\&lang=en

The quarterly time series of developed countries for the period 1995-2012 are adapted from "Quarterly National Accounts" by OECD database (2014). Retrieved from http://stats.oecd.org/BrandedView.aspx?oecd_bv_id=na-data-en\&doi=data-00017-en

the area of the triangle formed by the duration and amplitude of the cycle. This method reflects the idea of foregone output from peaks to troughs during contractions and output gains during expansion periods. We needed to compare these results with observations around the world. One possibility was to use the results of Calderón and Fuentes (2010) as referenced facts about business cycle durations and amplitudes in emerging and developed countries around the world. Although these results cover the two characteristics mentioned in the introduction, Calderón and Fuentes (2010) use data with a different range, excluding recent world financial crises. Therefore, we repeated the analysis with OECD quarterly data for the period 1995-2012. ${ }^{1}$ We divided countries into emerging and developed groups based on the definitions of Uribe and Schmitt-Grohé (2017). The developed countries are Australia, Austria, Belgium, Canada, Denmark, Finland, France, Germany, Hong Kong, Italy, Japan, Netherlands, Norway, Sweden, Switzerland, the United Kingdom and the United States. The emerging countries are Argentina, Israel, South Korea, Mexico, New Zealand, Peru, Portugal, South Africa, Spain, Turkey and Uruguay. The results are presented in Table 1.

We analyzed the cyclical properties of aggregate data series using annual GDP data, the final consumption expenditures of households and non-profit institutions serving households, the final consumption of the general government, gross capital formation, exports and imports of goods and services in millions of the national currency units at constant prices with reference to 2005, and population size. Aggregate data series of the nominal current account of the payment balance in millions of the national currency units were adjusted by the trade balance index volume with reference to 2005. For our analysis, we used data sets expressed intensively (per capita), i.e., divided by population size. Data from the period 1995-2013 were obtained from the Eurostat portal.

Macroeconomic textbooks such as Williamson (2014) and Uribe and Schmitt-Grohé (2017) use two approaches to measure the cyclical properties of aggregate data series according to Lucas's (1977) definition. The first approach is to compute the moments of cyclical components, and the second approach is to compute the moments of growth rates. The cyclical component time series of GDP (output; denoted by the letter $y$ ), household consumption (c), gross capital formation $(i)$, government consumption $(g)$, exports $(x)$ and imports $(m)$ is expressed as the difference between logs of corresponding intensively expressed observed and trend time series. The cyclical component time series of the trade balance $(t b)$, current account $(c a)$ and share of government consumption $(g y)$ are expressed 
Table 2. Moments of the Cyclical Components Computed Using the Log-Quadratic Trend

\begin{tabular}{|c|c|c|c|c|c|c|c|c|c|c|c|c|}
\hline $\begin{array}{l}\text { standard } \\
\text { deviations }\end{array}$ & BG & $C Z$ & $\mathrm{EE}$ & HR & $\mathrm{HU}$ & LV & LT & PL & RO & SK & SI & $\begin{array}{c}\text { weighted } \\
\text { average }\end{array}$ \\
\hline$\sigma_{y}$ & 6.51 & 4.51 & 6.19 & 4.06 & 3.14 & 7.90 & 6.43 & 2.14 & 15.12 & 4.44 & 3.51 & 5.92 \\
\hline$\sigma_{c} / \sigma_{y}$ & 1.19 & 0.69 & 1.31 & 1.13 & 1.44 & 1.37 & 1.38 & 0.89 & 0.71 & 0.84 & 0.74 & 0.94 \\
\hline$\sigma_{g} / \sigma_{y}$ & 1.35 & 0.70 & 0.65 & 0.69 & 1.19 & 0.55 & 0.46 & 1.45 & 0.28 & 0.91 & 0.58 & 0.98 \\
\hline$\sigma_{i} / \sigma_{y}$ & 3.69 & 2.06 & 3.50 & 3.56 & 2.24 & 2.59 & 3.22 & 5.61 & 1.18 & 3.00 & 3.58 & 3.50 \\
\hline$\sigma_{x} / \sigma_{y}$ & 1.61 & 1.17 & 1.51 & 1.27 & 1.76 & 0.86 & 1.37 & 2.25 & 0.43 & 1.62 & 1.88 & 1.54 \\
\hline$\sigma_{m} / \sigma_{y}$ & 1.88 & 1.06 & 2.08 & 2.26 & 1.71 & 1.72 & 1.85 & 3.73 & 0.87 & 1.51 & 2.15 & 2.25 \\
\hline$\sigma_{t b / y}$ & 5.17 & 1.23 & 5.02 & 2.76 & 1.23 & 5.95 & 4.09 & 1.87 & 4.93 & 2.40 & 1.47 & 2.86 \\
\hline$\sigma_{\text {caly }}$ & 10.43 & 2.57 & 17.08 & - & 1.72 & 10.83 & 59.76 & - & - & 19.18 & 3.33 & - \\
\hline $\begin{array}{l}\text { correlations } \\
\text { with y }\end{array}$ & BG & $C Z$ & EE & $H R$ & HU & LV & LT & PL & RO & SK & SI & $\begin{array}{c}\text { weighted } \\
\text { average }\end{array}$ \\
\hline c & 0.94 & 0.90 & 0.95 & 0.96 & 0.90 & 0.97 & 0.96 & 0.88 & 0.15 & 0.92 & 0.76 & 0.75 \\
\hline i & 0.81 & 0.94 & 0.93 & 0.92 & 0.53 & 0.89 & 0.83 & 0.95 & -0.02 & 0.78 & 0.96 & 0.69 \\
\hline$x$ & 0.18 & 0.71 & 0.63 & 0.72 & 0.39 & 0.73 & 0.66 & 0.39 & -0.24 & 0.49 & 0.91 & 0.33 \\
\hline$m$ & 0.60 & 0.67 & 0.88 & 0.92 & 0.43 & 0.89 & 0.88 & 0.85 & -0.01 & 0.66 & 0.96 & 0.60 \\
\hline tb & -0.85 & 0.40 & -0.94 & -0.86 & -0.41 & -0.92 & -0.89 & -0.89 & -0.14 & -0.26 & -0.69 & -0.53 \\
\hline$c a$ & -0.76 & -0.08 & -0.28 & - & -0.15 & -0.93 & -0.23 & - & - & 0.07 & -0.83 & - \\
\hline gy & -0.05 & -0.76 & -0.61 & -0.68 & -0.02 & -0.78 & -0.91 & -0.17 & -0.96 & -0.52 & -0.81 & -0.46 \\
\hline $\begin{array}{l}\text { serial } \\
\text { correlations }\end{array}$ & BG & $C Z$ & EE & HR & $\mathrm{HU}$ & LV & LT & PL & RO & SK & SI & $\begin{array}{c}\text { weighted } \\
\text { average }\end{array}$ \\
\hline$y$ & 0.70 & 0.78 & 0.57 & 0.72 & 0.63 & 0.66 & 0.61 & 0.67 & -0.17 & 0.72 & 0.61 & 0.51 \\
\hline c & 0.71 & 0.77 & 0.70 & 0.60 & 0.65 & 0.67 & 0.71 & 0.65 & 0.80 & 0.76 & 0.61 & 0.70 \\
\hline$g$ & 0.20 & 0.49 & 0.72 & 0.69 & 0.65 & 0.45 & 0.66 & 0.75 & -0.07 & 0.38 & 0.64 & 0.48 \\
\hline$i$ & 0.22 & 0.56 & 0.50 & 0.61 & -0.05 & 0.49 & 0.22 & 0.61 & 0.70 & 0.30 & 0.54 & 0.50 \\
\hline$x$ & 0.11 & 0.31 & 0.28 & 0.36 & 0.40 & 0.37 & 0.35 & 0.18 & 0.46 & 0.30 & 0.46 & 0.29 \\
\hline$m$ & 0.24 & 0.23 & 0.41 & 0.55 & 0.21 & 0.50 & 0.44 & 0.42 & 0.66 & 0.30 & 0.49 & 0.42 \\
\hline tb & 0.61 & 0.45 & 0.64 & 0.45 & 0.34 & 0.51 & 0.53 & 0.62 & 0.63 & 0.04 & 0.42 & 0.53 \\
\hline $\mathrm{ca}$ & 0.70 & 0.04 & -0.40 & - & -0.09 & 0.49 & -0.20 & - & - & -0.02 & 0.09 & - \\
\hline Average & BG & $C Z$ & $\mathrm{EE}$ & HR & $\mathrm{HU}$ & LV & LT & PL & RO & SK & SI & $\begin{array}{c}\text { weighted } \\
\text { average }\end{array}$ \\
\hline$(x+m) / y$ & 113.22 & 119.32 & 147.97 & 83.79 & 134.68 & 108.74 & 118.39 & 69.93 & 71.90 & 145.73 & 119.78 & 93.07 \\
\hline
\end{tabular}

Note: The annual time series of post-communist countries for the period 1995-2013 are adapted from "GDP and main components (output, expenditure and income)" by Eurostat database (2014). Retrieved from http://appsso.eurostat.ec.europa.eu/ nui/show.do?dataset=nama_10_gdp\&lang=en 
Table 3. Moments of Cyclical Components - the Hodrick-Prescott Filter $(\lambda=100)$

\begin{tabular}{|c|c|c|c|c|c|c|c|c|c|c|c|c|}
\hline $\begin{array}{l}\text { standard } \\
\text { deviations }\end{array}$ & BG & $C Z$ & $\mathrm{EE}$ & HR & HU & LV & LT & PL & RO & SK & SI & $\begin{array}{c}\text { weighted } \\
\text { average }\end{array}$ \\
\hline$\sigma_{y}$ & 5.15 & 3.59 & 6.07 & 3.86 & 2.89 & 7.31 & 5.84 & 2.04 & 15.22 & 3.73 & 3.44 & 5.62 \\
\hline$\sigma_{c} / \sigma_{y}$ & 1.22 & 0.72 & 1.28 & 1.12 & 1.44 & 1.34 & 1.34 & 0.99 & 0.55 & 0.89 & 0.74 & 0.95 \\
\hline$\sigma_{g} / \sigma_{y}$ & 1.57 & 0.82 & 0.54 & 0.63 & 1.10 & 0.60 & 0.44 & 1.24 & 0.28 & 1.00 & 0.60 & 0.92 \\
\hline$\sigma_{i} / \sigma_{y}$ & 4.37 & 2.23 & 3.46 & 3.62 & 2.77 & 2.76 & 3.53 & 5.55 & 0.95 & 3.43 & 3.67 & 3.59 \\
\hline$\sigma_{x} / \sigma_{y}$ & 1.99 & 1.44 & 1.51 & 1.42 & 2.09 & 0.91 & 1.47 & 2.31 & 0.38 & 1.85 & 1.78 & 1.66 \\
\hline$\sigma_{m} / \sigma_{y}$ & 2.30 & 1.34 & 2.08 & 2.30 & 2.23 & 1.80 & 1.94 & 4.03 & 0.72 & 1.82 & 2.08 & 2.45 \\
\hline$\sigma_{t b / y}$ & 4.65 & 1.21 & 4.68 & 2.51 & 1.39 & 5.59 & 3.80 & 1.80 & 4.35 & 2.59 & 1.49 & 2.67 \\
\hline$\sigma_{c a / y}$ & 9.23 & 2.55 & 16.61 & - & 1.77 & 10.22 & 59.21 & - & - & 18.36 & 3.14 & - \\
\hline $\begin{array}{l}\text { correlations } \\
\text { with } y\end{array}$ & BG & $\mathrm{CZ}$ & $\mathrm{EE}$ & HR & $\mathrm{HU}$ & LV & LT & PL & RO & SK & SI & $\begin{array}{c}\text { weighted } \\
\text { average }\end{array}$ \\
\hline c & 0.90 & 0.85 & 0.95 & 0.95 & 0.89 & 0.97 & 0.95 & 0.87 & 0.22 & 0.86 & 0.77 & 0.75 \\
\hline i & 0.76 & 0.92 & 0.94 & 0.92 & 0.65 & 0.89 & 0.84 & 0.95 & 0.02 & 0.77 & 0.97 & 0.70 \\
\hline$x$ & 0.16 & 0.70 & 0.70 & 0.79 & 0.52 & 0.74 & 0.64 & 0.47 & -0.15 & 0.48 & 0.88 & 0.38 \\
\hline$m$ & 0.55 & 0.67 & 0.90 & 0.93 & 0.59 & 0.89 & 0.88 & 0.86 & 0.08 & 0.67 & 0.94 & 0.63 \\
\hline$t b$ & -0.78 & 0.31 & -0.91 & -0.79 & -0.53 & -0.91 & -0.87 & -0.91 & -0.19 & -0.29 & -0.70 & -0.56 \\
\hline$c a$ & -0.67 & -0.13 & -0.29 & - & -0.22 & -0.93 & -0.23 & - & - & -0.05 & -0.79 & - \\
\hline gy & 0.10 & -0.73 & -0.75 & -0.75 & -0.26 & -0.74 & -0.90 & -0.31 & -0.97 & -0.49 & -0.79 & -0.52 \\
\hline $\begin{array}{l}\text { serial } \\
\text { correlations }\end{array}$ & BG & $\mathrm{CZ}$ & $\mathrm{EE}$ & HR & $\mathrm{HU}$ & LV & LT & PL & RO & SK & SI & $\begin{array}{c}\text { weighted } \\
\text { average }\end{array}$ \\
\hline$y$ & 0.63 & 0.72 & 0.58 & 0.69 & 0.69 & 0.65 & 0.57 & 0.63 & -0.27 & 0.65 & 0.61 & 0.46 \\
\hline c & 0.65 & 0.68 & 0.70 & 0.56 & 0.75 & 0.63 & 0.67 & 0.58 & 0.75 & 0.69 & 0.57 & 0.66 \\
\hline$g$ & 0.19 & 0.50 & 0.64 & 0.65 & 0.63 & 0.50 & 0.60 & 0.69 & -0.09 & 0.27 & 0.64 & 0.44 \\
\hline i & 0.29 & 0.44 & 0.50 & 0.55 & 0.20 & 0.51 & 0.20 & 0.58 & 0.62 & 0.21 & 0.56 & 0.48 \\
\hline$x$ & 0.08 & 0.32 & 0.27 & 0.42 & 0.48 & 0.33 & 0.33 & 0.13 & 0.42 & 0.30 & 0.41 & 0.27 \\
\hline$m$ & 0.24 & 0.24 & 0.41 & 0.53 & 0.45 & 0.46 & 0.39 & 0.38 & 0.59 & 0.29 & 0.47 & 0.41 \\
\hline tb & 0.63 & 0.44 & 0.62 & 0.36 & 0.59 & 0.47 & 0.48 & 0.58 & 0.56 & 0.03 & 0.42 & 0.52 \\
\hline $\mathrm{ca}$ & 0.67 & 0.03 & -0.38 & - & -0.05 & 0.45 & -0.22 & - & - & -0.07 & -0.01 & - \\
\hline average & BG & $C Z$ & EE & HR & HU & LV & LT & PL & RO & SK & SI & $\begin{array}{c}\text { weighted } \\
\text { average }\end{array}$ \\
\hline$(x+m) / y$ & 113.22 & 119.32 & 147.97 & 83.79 & 134.68 & 108.74 & 118.39 & 69.93 & 71.90 & 145.73 & 119.78 & 93.07 \\
\hline
\end{tabular}

Note: The value $\lambda=100$ used in these calculations is suggested by Hodrick and Prescott (1997).

The annual time series of post-communist countries for the period 1995-2013 are adapted from "GDP and main components (output, expenditure and income)"by Eurostat database (2014). Retrieved from http://appsso.eurostat.ec.europa.eu/nui/show. do?dataset=nama_10_gdp\&lang=en 
Table 4. Moments of Cyclical Components - the Hodrick-Prescott Filter $(\lambda=6.25)$

\begin{tabular}{|c|c|c|c|c|c|c|c|c|c|c|c|c|}
\hline $\begin{array}{l}\text { standard } \\
\text { deviations }\end{array}$ & BG & $C Z$ & EE & HR & $\mathrm{HU}$ & LV & LT & PL & RO & SK & SI & $\begin{array}{c}\text { weighted } \\
\text { average }\end{array}$ \\
\hline$\sigma_{y}$ & 2.41 & 1.69 & 4.25 & 2.09 & 1.45 & 4.73 & 3.97 & 1.12 & 12.43 & 2.09 & 1.95 & 3.88 \\
\hline$\sigma_{c} / \sigma_{y}$ & 1.37 & 0.73 & 1.13 & 1.31 & 1.30 & 1.38 & 1.21 & 0.87 & 0.29 & 0.78 & 0.64 & 0.85 \\
\hline$\sigma_{g} / \sigma_{y}$ & 2.44 & 1.15 & 0.43 & 0.65 & 1.13 & 0.70 & 0.41 & 1.05 & 0.30 & 1.34 & 0.48 & 0.97 \\
\hline$\sigma_{i} / \sigma_{y}$ & 7.07 & 3.25 & 3.71 & 4.50 & 4.14 & 3.05 & 4.28 & 6.18 & 0.66 & 4.83 & 4.10 & 4.33 \\
\hline$\sigma_{x} / \sigma_{y}$ & 3.75 & 2.41 & 1.79 & 1.77 & 2.98 & 1.13 & 1.78 & 3.27 & 0.30 & 2.73 & 2.28 & 2.38 \\
\hline$\sigma_{m} / \sigma_{y}$ & 4.03 & 2.27 & 2.32 & 2.86 & 3.10 & 2.11 & 2.21 & 4.83 & 0.50 & 2.58 & 2.52 & 3.08 \\
\hline$\sigma_{t b / y}$ & 2.91 & 0.95 & 3.13 & 1.88 & 0.86 & 3.94 & 2.51 & 0.98 & 2.61 & 2.12 & 1.05 & 1.67 \\
\hline$\sigma_{\text {caly }}$ & 5.55 & 2.31 & 15.04 & - & 1.56 & 7.50 & 54.99 & - & - & 15.66 & 2.51 & - \\
\hline $\begin{array}{l}\text { correlations } \\
\text { with y }\end{array}$ & BG & $C Z$ & EE & HR & HU & LV & LT & PL & RO & SK & SI & $\begin{array}{c}\text { weighted } \\
\text { average }\end{array}$ \\
\hline c & 0.66 & 0.53 & 0.93 & 0.91 & 0.67 & 0.95 & 0.92 & 0.78 & 0.46 & 0.72 & 0.54 & 0.68 \\
\hline i & 0.82 & 0.92 & 0.96 & 0.88 & 0.76 & 0.85 & 0.86 & 0.93 & 0.15 & 0.75 & 0.95 & 0.73 \\
\hline$x$ & 0.26 & 0.77 & 0.76 & 0.76 & 0.70 & 0.75 & 0.65 & 0.58 & 0.04 & 0.57 & 0.88 & 0.50 \\
\hline$m$ & 0.60 & 0.83 & 0.94 & 0.92 & 0.77 & 0.87 & 0.86 & 0.82 & 0.14 & 0.81 & 0.94 & 0.67 \\
\hline tb & -0.75 & 0.20 & -0.88 & -0.70 & -0.29 & -0.84 & -0.83 & -0.85 & -0.14 & -0.22 & -0.47 & -0.50 \\
\hline$c a$ & -0.61 & -0.37 & -0.29 & - & -0.01 & -0.92 & -0.18 & - & - & -0.46 & -0.78 & - \\
\hline gy & 0.53 & -0.80 & -0.87 & -0.80 & -0.55 & -0.60 & -0.91 & -0.56 & -0.98 & -0.49 & -0.91 & -0.61 \\
\hline $\begin{array}{l}\text { serial } \\
\text { correlations }\end{array}$ & BG & $C Z$ & EE & HR & HU & LV & LT & PL & RO & SK & SI & $\begin{array}{c}\text { weighted } \\
\text { average }\end{array}$ \\
\hline$y$ & 0.07 & 0.25 & 0.37 & 0.29 & 0.13 & 0.44 & 0.29 & 0.40 & -0.33 & 0.27 & 0.17 & 0.17 \\
\hline c & 0.25 & 0.09 & 0.51 & 0.17 & 0.23 & 0.41 & 0.41 & 0.31 & 0.21 & 0.32 & 0.06 & 0.25 \\
\hline$g$ & -0.08 & 0.14 & 0.25 & 0.22 & 0.10 & 0.28 & 0.27 & 0.15 & -0.20 & -0.07 & 0.13 & 0.06 \\
\hline i & -0.02 & 0.05 & 0.30 & 0.27 & -0.24 & 0.26 & -0.01 & 0.36 & 0.20 & -0.03 & 0.17 & 0.17 \\
\hline$x$ & -0.08 & 0.09 & 0.09 & -0.07 & 0.23 & 0.17 & 0.16 & -0.27 & -0.15 & 0.15 & 0.09 & -0.09 \\
\hline$m$ & 0.01 & -0.03 & 0.22 & 0.18 & 0.08 & 0.27 & 0.16 & 0.07 & 0.08 & 0.07 & 0.11 & 0.07 \\
\hline tb & 0.37 & 0.30 & 0.43 & 0.04 & 0.10 & 0.21 & 0.09 & 0.31 & 0.20 & -0.03 & 0.10 & 0.23 \\
\hline$c a$ & 0.45 & -0.10 & -0.63 & - & -0.22 & 0.24 & -0.32 & - & - & -0.27 & -0.31 & - \\
\hline average & BG & $C Z$ & EE & HR & HU & LV & LT & PL & RO & SK & SI & $\begin{array}{c}\text { weighted } \\
\text { average }\end{array}$ \\
\hline$(x+m) / y$ & 113.22 & 119.32 & 147.97 & 83.79 & 134.68 & 108.74 & 118.39 & 69.93 & 71.90 & 145.73 & 119.78 & 93.07 \\
\hline
\end{tabular}

Note: The value $\lambda=6.25$ used in these calculations is suggested by Ravn and Uhlig (2002).

The annual time series of post-communist countries for the period 1995-2013 are adapted from "GDP and main components (output, expenditure and income)"by Eurostat database (2014). Retrieved from http://appsso.eurostat.ec.europa.eu/nui/show. do?dataset=nama_10_gdp\&lang=en 
Table 5. Moments of Growth Rates

\begin{tabular}{|c|c|c|c|c|c|c|c|c|c|c|c|c|}
\hline $\begin{array}{l}\text { standard } \\
\text { deviations }\end{array}$ & BG & $C Z$ & $\mathrm{EE}$ & HR & $\mathrm{HU}$ & LV & LT & PL & RO & SK & SI & $\begin{array}{c}\text { weighted } \\
\text { average }\end{array}$ \\
\hline$\sigma_{y}$ & 4.65 & 3.08 & 6.34 & 4.00 & 2.89 & 6.84 & 5.89 & 1.86 & 21.26 & 3.37 & 3.76 & 6.67 \\
\hline$\sigma_{c} / \sigma_{y}$ & 1.23 & 0.78 & 1.14 & 1.19 & 1.35 & 1.34 & 1.20 & 1.12 & 0.32 & 0.92 & 0.74 & 0.95 \\
\hline$\sigma_{g} / \sigma_{y}$ & 2.04 & 1.04 & 0.48 & 0.58 & 1.03 & 0.71 & 0.42 & 1.17 & 0.30 & 1.36 & 0.60 & 0.96 \\
\hline$\sigma_{i} / \sigma_{y}$ & 5.80 & 2.92 & 3.60 & 3.81 & 3.97 & 3.27 & 4.66 & 5.75 & 0.64 & 4.83 & 3.75 & 4.01 \\
\hline$\sigma_{x} / \sigma_{y}$ & 3.10 & 2.13 & 1.84 & 1.77 & 2.46 & 1.15 & 1.74 & 3.58 & 0.31 & 2.50 & 1.91 & 2.35 \\
\hline$\sigma_{m} / \sigma_{y}$ & 3.28 & 2.12 & 2.31 & 2.51 & 2.83 & 2.12 & 2.23 & 5.15 & 0.52 & 2.57 & 2.18 & 3.08 \\
\hline$\sigma_{t b / y}$ & 4.68 & 1.17 & 4.73 & 2.58 & 1.25 & 4.82 & 3.63 & 1.81 & 4.52 & 2.35 & 1.46 & 2.66 \\
\hline$\sigma_{c a / y}$ & 9.47 & 2.63 & 17.27 & - & 1.69 & 9.52 & 61.78 & - & - & 19.01 & 3.11 & - \\
\hline $\begin{array}{l}\text { correlations } \\
\text { with y }\end{array}$ & BG & $C Z$ & $\mathrm{EE}$ & HR & $\mathrm{HU}$ & LV & LT & PL & RO & SK & SI & $\begin{array}{c}\text { weighted } \\
\text { average }\end{array}$ \\
\hline c & 0.69 & 0.72 & 0.92 & 0.91 & 0.81 & 0.93 & 0.92 & 0.80 & 0.29 & 0.70 & 0.75 & 0.69 \\
\hline i & 0.78 & 0.90 & 0.95 & 0.88 & 0.77 & 0.83 & 0.85 & 0.93 & 0.02 & 0.76 & 0.96 & 0.70 \\
\hline$x$ & 0.23 & 0.73 & 0.75 & 0.81 & 0.73 & 0.71 & 0.64 & 0.61 & -0.02 & 0.58 & 0.84 & 0.49 \\
\hline$m$ & 0.51 & 0.76 & 0.91 & 0.90 & 0.79 & 0.84 & 0.86 & 0.84 & 0.09 & 0.77 & 0.93 & 0.66 \\
\hline tb & -0.22 & 0.16 & -0.34 & -0.06 & -0.42 & -0.47 & -0.21 & -0.36 & -0.16 & -0.22 & -0.07 & -0.24 \\
\hline ca & -0.01 & -0.01 & -0.34 & - & -0.17 & -0.52 & -0.11 & - & - & -0.66 & -0.26 & - \\
\hline gy & 0.54 & -0.24 & -0.71 & -0.59 & -0.13 & -0.51 & -0.62 & 0.13 & -0.60 & -0.41 & -0.52 & -0.16 \\
\hline $\begin{array}{l}\text { serial } \\
\text { correlations }\end{array}$ & BG & $C Z$ & $\mathrm{EE}$ & HR & HU & LV & LT & PL & RO & SK & SI & $\begin{array}{c}\text { weighted } \\
\text { average }\end{array}$ \\
\hline$y$ & 0.47 & 0.42 & 0.39 & 0.57 & 0.38 & 0.45 & 0.23 & 0.45 & -0.35 & 0.28 & 0.35 & 0.27 \\
\hline c & 0.37 & 0.46 & 0.62 & 0.32 & 0.65 & 0.39 & 0.41 & 0.57 & 0.32 & 0.53 & 0.43 & 0.48 \\
\hline$g$ & 0.26 & 0.07 & 0.32 & 0.32 & 0.16 & 0.29 & 0.15 & 0.20 & -0.36 & -0.16 & 0.53 & 0.07 \\
\hline i & 0.19 & 0.02 & 0.26 & 0.38 & -0.15 & 0.25 & -0.11 & 0.36 & 0.26 & -0.05 & 0.29 & 0.21 \\
\hline$x$ & -0.25 & 0.03 & -0.06 & 0.09 & 0.25 & 0.04 & 0.02 & -0.31 & -0.14 & 0.03 & 0.01 & -0.13 \\
\hline$m$ & -0.08 & -0.02 & 0.09 & 0.25 & 0.22 & 0.18 & 0.01 & 0.08 & 0.17 & -0.06 & 0.12 & 0.09 \\
\hline tb & 0.59 & 0.43 & 0.64 & 0.43 & 0.31 & 0.44 & 0.52 & 0.62 & 0.65 & 0.04 & 0.41 & 0.53 \\
\hline $\mathrm{ca}$ & 0.71 & 0.05 & -0.39 & - & -0.10 & 0.44 & -0.20 & - & - & -0.02 & 0.10 & - \\
\hline average & BG & $C Z$ & EE & HR & $\mathrm{HU}$ & LV & LT & PL & RO & SK & SI & $\begin{array}{c}\text { weighted } \\
\text { average }\end{array}$ \\
\hline$(x+m) / y$ & 113.22 & 119.32 & 147.97 & 83.79 & 134.68 & 108.74 & 118.39 & 69.93 & 71.90 & 145.73 & 119.78 & 93.07 \\
\hline
\end{tabular}

Note: The annual time series of post-communist countries for the period 1995-2013 are adapted from "GDP and main components (output, expenditure and income)"by Eurostat database (2014). Retrieved from http://appsso.eurostat.ec.europa.eu/ nui/show.do?dataset=nama_10_gdp\&lang=en 

methods roughly generate the same results. Therefore, we used only one approach in our further analysis. To compare our results with other analyses in the literature, we chose a log-quadratic trend to compute cyclical components.

In Table 6, we present a comparison of the average moments of cyclical components using a log-quadratic trend of 11 post-communist, emerging, and developed countries (i.e., the last column of Table 2). The results for poor, emerging, and developed countries are obtained from Uribe and Schmitt-Grohé (2017), who used data from the period 1965-2010 and employed the same methodology.

It follows from Table 6 that the aggregate economic activity of 11 post-communist countries in the period 1995-2013 was less volatile than the aggregate economic activity of emerging countries in the period 1965-2010. Notice that this period includes the shorter period of 1982-2008, which has been labeled by many authors (e.g., Williamson, 2014) as "the Great Moderation" because U.S. output was less volatile in this period. The post-communist trade balance is relatively strongly counter-cyclical, contradicting the argument in the introduction that the trade balance-to-output ratio is more negatively correlated with output as the level of economic development increases. Post-communist countries are similar to other emerging countries in terms of the volatility of consumption.

For a more detailed view, we should divide emerging countries according to population size because economic activity in larger countries is less volatile. We should compare the cyclical component moments of similarly sized countries. As stated earlier, using the definition of Uribe and Schmitt-Grohé (2017), Poland and Romania are medium-sized countries. The weighted averages of the moments of cyclical components in small countries (i.e., 9 small post-communist countries in the period 1995-2013) are computed in Table 7. The results for poor, emerging, and developed countries are obtained from Uribe and Schmitt-Grohé (2017), who used data from the period 1965-2010 and employed the same methodology.

The results of the analysis are similar: output volatility in post-communist countries in the period 1995-2013 is lower than that in poor and emerging countries from 1965-2010. The volatility of govern- ment share of GDP in post-communist countries (1995-2013) is similar to that in developed countries (1965-2010). Consumption is highly volatile and the trade balance or current account is more strongly counter-cyclical in post-communist countries (1995-2013).

We reach the same conclusions if we compare the results of Poland with those of other medium-sized countries (see Table 8). In contrast, the results for Romania are unique. Romanian output is very volatile and the trade balance is weakly counter-cyclical. Furthermore, output in Romania is negatively serially correlated. We can conclude that Romanian business cycles differ from those of other post-communist countries and that this analysis is less helpful in the case of Romania than it is in the cases of other postcommunist countries.

Assisted by other studies, we can put forth several observations from this "first look at the data" as our contribution to the discussion of results.

We cannot overlook the long-term development of post-communist countries. Szomolányi, Lukáčiková and Lukáčik (2011) show that post-communist countries were in a transitional state in the period 1997-2009 and that they were converging to a higher level expressed by steady state GDP per capita. The steady state was similar for European Union countries. ${ }^{2}$ In other words, post-communist European Union members may be as rich as Germany several decades from now. It follows from neoclassical growth theory that as post-communist countries have been in a transitional state, their steady state has changed (risen) dramatically. This change stems from events such as economic system transformation, opening up to international markets, price liberalization, privatization, tax reformation and legal system transformation, among others. All these events have been present in some form in all post-communist countries during the period studied. Moreover, there were currency transformations in many countries. As explained by neoclassical growth theory, events causing changes in steady states are themselves mostly caused by permanent real shocks. Indeed, the afore-mentioned events have the character of permanent real shocks, which may explain why post-communist business cycles last longer than those of other countries (see Table 1). It follows from the real business cycle principles 
Table 6. Cyclical Properties of Post-Communist, Poor, Emerging and Developed Countries

\begin{tabular}{|c|c|c|c|c|}
\hline \multirow[b]{2}{*}{ standard deviations } & \multicolumn{4}{|c|}{ Countries } \\
\hline & post-communist & poor & emerging & developed \\
\hline$\sigma_{y}$ & 5.92 & 6.08 & 8.71 & 3.32 \\
\hline$\sigma_{c} / \sigma_{y}$ & 0.94 & 1.12 & 0.98 & 0.87 \\
\hline$\sigma_{g} / \sigma_{y}$ & 0.98 & 2.46 & 2.00 & 1.73 \\
\hline$\sigma_{i} / \sigma_{y}$ & 3.50 & 3.24 & 2.79 & 3.20 \\
\hline$\sigma_{x} / \sigma_{y}$ & 1.54 & 3.08 & 2.82 & 3.36 \\
\hline$\sigma_{m} / \sigma_{y}$ & 2.25 & 3.30 & 2.72 & 3.64 \\
\hline$\sigma_{t b / y}$ & 2.86 & 2.12 & 3.80 & 1.25 \\
\hline$\sigma_{\text {caly }}$ & - & 2.06 & 3.08 & 1.39 \\
\hline correlations with $y$ & post-communist & poor & emerging & developed \\
\hline c & 0.75 & 0.66 & 0.75 & 0.76 \\
\hline i & 0.69 & 0.60 & 0.77 & 0.77 \\
\hline$x$ & 0.33 & 0.14 & 0.35 & 0.17 \\
\hline$m$ & 0.60 & 0.14 & 0.50 & 0.34 \\
\hline tb & -0.53 & -0.11 & -0.21 & -0.26 \\
\hline$c a$ & - & -0.28 & -0.24 & -0.30 \\
\hline gy & -0.46 & 0.08 & -0.08 & -0.39 \\
\hline serial correlations & post-communist & poor & emerging & developed \\
\hline$y$ & 0.51 & 0.65 & 0.87 & 0.76 \\
\hline c & 0.70 & 0.62 & 0.74 & 0.75 \\
\hline$g$ & 0.48 & 0.71 & 0.80 & 0.89 \\
\hline i & 0.50 & 0.49 & 0.72 & 0.67 \\
\hline$x$ & 0.29 & 0.65 & 0.74 & 0.74 \\
\hline$m$ & 0.42 & 0.61 & 0.74 & 0.69 \\
\hline tb & 0.53 & 0.59 & 0.62 & 0.69 \\
\hline$c a$ & - & 0.55 & 0.52 & 0.71 \\
\hline average & post-communist & poor & emerging & developed \\
\hline$(x+m) / y$ & 93.07 & 32.50 & 46.40 & 40.40 \\
\hline
\end{tabular}

Source: Uribe and Schmitt-Grohé (2017) and authors' calculations.

Note: The annual time series of post-communist countries for the period 1995-2013 are adapted from "GDP and main components (output, expenditure and income)" by Eurostat database (2014). Retrieved from http://appsso.eurostat.ec.europa.eu/ nui/show.do?dataset=nama_10_gdp\&lang=en 
Table 7. Cyclical Properties of Small Countries

\begin{tabular}{|c|c|c|c|c|}
\hline \multirow[b]{2}{*}{ standard deviations } & \multicolumn{4}{|c|}{ small countries } \\
\hline & post-communist & poor & emerging & developed \\
\hline$\sigma_{y}$ & 4.80 & 8.17 & 9.50 & 4.31 \\
\hline$\sigma_{c} / \sigma_{y}$ & 1.09 & 1.39 & 0.97 & 0.92 \\
\hline$\sigma_{g} / \sigma_{y}$ & 0.91 & 2.92 & 1.85 & 1.66 \\
\hline$\sigma_{i} / \sigma_{y}$ & 2.83 & 4.68 & 2.97 & 3.07 \\
\hline$\sigma_{x} / \sigma_{y}$ & 1.47 & 2.81 & 2.23 & 2.23 \\
\hline$\sigma_{m} / \sigma_{y}$ & 1.66 & 2.96 & 2.25 & 2.36 \\
\hline$\sigma_{t b / y}$ & 2.72 & 5.62 & 4.00 & 2.29 \\
\hline$\sigma_{c a l y}$ & 10.47 & 4.84 & 3.55 & 2.37 \\
\hline correlation with $y$ & post-communist & poor & emerging & developed \\
\hline$c$ & 0.92 & 0.58 & 0.73 & 0.55 \\
\hline$i$ & 0.80 & 0.45 & 0.72 & 0.63 \\
\hline$x$ & 0.53 & 0.53 & 0.53 & 0.58 \\
\hline$m$ & 0.67 & 0.53 & 0.62 & 0.63 \\
\hline tb & -0.42 & -0.04 & -0.21 & -0.11 \\
\hline $\mathrm{ca}$ & -0.31 & -0.17 & -0.20 & -0.11 \\
\hline gy & -0.46 & 0.02 & 0.03 & -0.26 \\
\hline serial correlations & post-communist & poor & emerging & developed \\
\hline$y$ & 0.69 & 0.76 & 0.89 & 0.83 \\
\hline c & 0.70 & 0.61 & 0.70 & 0.73 \\
\hline$g$ & 0.51 & 0.61 & 0.78 & 0.87 \\
\hline i & 0.32 & 0.62 & 0.67 & 0.71 \\
\hline$x$ & 0.31 & 0.58 & 0.74 & 0.68 \\
\hline$m$ & 0.31 & 0.68 & 0.71 & 0.66 \\
\hline tb & 0.42 & 0.50 & 0.52 & 0.67 \\
\hline$c a$ & 0.13 & 0.36 & 0.40 & 0.56 \\
\hline average & post-communist & poor & emerging & developed \\
\hline$(x+m) / y$ & 121.60 & 57.70 & 69.20 & 116.80 \\
\hline
\end{tabular}

Source: Uribe and Schmitt-Grohé (2017) and authors' calculations.

Note: The annual time series of post-communist countries for the period 1995-2013 are adapted from "GDP and main components (output, expenditure and income)" by Eurostat database (2014). Retrieved from http://appsso.eurostat.ec.europa.eu/ nui/show.do?dataset=nama_10_gdp\&lang=en 
Table 8. Cyclical Properties of Medium-sized Countries

\begin{tabular}{|c|c|c|c|c|c|}
\hline \multirow[b]{2}{*}{ standard deviations } & \multicolumn{5}{|c|}{ medium-sized countries } \\
\hline & Poland & Romania & poor & emerging & developed \\
\hline$\sigma_{y}$ & 2.14 & 15.12 & 9.46 & 8.99 & 3.05 \\
\hline$\sigma_{c} / \sigma_{y}$ & 0.89 & 0.71 & 1.05 & 0.93 & 0.93 \\
\hline$\sigma_{g} / \sigma_{y}$ & 1.45 & 0.28 & 2.86 & 2.05 & 1.71 \\
\hline$\sigma_{i} / \sigma_{y}$ & 5.60 & 1.18 & 4.01 & 2.86 & 3.07 \\
\hline$\sigma_{x} / \sigma_{y}$ & 2.25 & 0.43 & 3.94 & 2.92 & 3.33 \\
\hline$\sigma_{m} / \sigma_{y}$ & 3.73 & 0.87 & 3.45 & 2.68 & 3.80 \\
\hline$\sigma_{t b / y}$ & 1.87 & 4.93 & 3.82 & 4.39 & 1.47 \\
\hline$\sigma_{c a l y}$ & - & - & 3.40 & 3.45 & 1.47 \\
\hline correlation with $y$ & Poland & Romania & poor & emerging & developed \\
\hline c & 0.88 & 0.15 & 0.74 & 0.70 & 0.70 \\
\hline i & 0.95 & -0.02 & 0.55 & 0.76 & 0.74 \\
\hline$x$ & 0.39 & -0.24 & 0.58 & 0.36 & 0.37 \\
\hline$m$ & 0.85 & -0.01 & 0.62 & 0.57 & 0.47 \\
\hline tb & -0.89 & -0.14 & -0.25 & -0.24 & -0.24 \\
\hline$c a$ & - & - & -0.11 & -0.34 & -0.08 \\
\hline gy & -0.17 & -0.96 & 0.24 & 0.00 & -0.40 \\
\hline serial correlations & Poland & Romania & poor & emerging & developed \\
\hline$y$ & 0.67 & -0.17 & 0.84 & 0.84 & 0.80 \\
\hline c & 0.65 & 0.80 & 0.61 & 0.71 & 0.75 \\
\hline$g$ & 0.75 & -0.07 & 0.74 & 0.80 & 0.89 \\
\hline i & 0.61 & 0.70 & 0.64 & 0.70 & 0.61 \\
\hline$x$ & 0.18 & 0.46 & 0.73 & 0.76 & 0.75 \\
\hline$m$ & 0.42 & 0.66 & 0.68 & 0.72 & 0.71 \\
\hline tb & 0.62 & 0.63 & 0.51 & 0.58 & 0.68 \\
\hline$c a$ & - & - & 0.42 & 0.46 & 0.67 \\
\hline average & Poland & Romania & poor & emerging & developed \\
\hline$(x+m) / y$ & 69.93 & 71.90 & 48.90 & 49.70 & 45.20 \\
\hline
\end{tabular}

Source: Uribe and Schmitt-Grohé (2017) and authors' calculations.

Note: The annual time series of Poland and Romania for the period 1995-2013 are adapted from "GDP and main components (output, expenditure and income)"by Eurostat database (2014). Retrieved from http://appsso.eurostat.ec.europa.eu/nui/show. do?dataset=nama_10_gdp\&lang=en 
outlined in the introduction that higher consumption volatility and trade balance (current account) counter-cyclicality occur if permanent real shocks occur.

García-Cicco et al. (2010), Uribe and Yue (2006) and Uribe and Schmitt-Grohé (2017) state that the business cycles of emerging countries are driven by interest rate and interest rate spread shocks, but our study cannot confirm that financial friction is an important determinant of the business cycle in post-communist countries. Consumption is relatively highly volatile in post-communist countries, but their trade balance is too counter-cyclical. For example, Szomolányi et al. (2014a; 2014b) conducted SVAR analysis that shows that interest rate and interest rate spread shocks have had a very small impact on Slovak and Czech business cycles.

Financial friction is a significant factor in the short- and medium-term economic performance of emerging and poor countries around the world. As the credibility of domestic economic policy decreases, domestic interest rate spreads increase. Low output is one of the consequences of decreased policy credibility. The limited ability of agents to borrow causes a cumulative fall in output. Interest rate and interest rate spread shocks cause high domestic output volatility. Therefore, output volatility is higher in emerging and poor countries around the world than in postcommunist countries.

\section{Conclusion}

Even if we consider European post-communist countries to be emerging, their business cycle characteristics differ slightly from those of emerging countries around the world:

- post-communist countries' expansions and recessions are longer.

- post-communist countries' recessions are more pronounced.

- post-communist countries' output is relatively low in volatility.

- post-communist countries' household consumption is relatively highly volatile.

- the government share of GDP is countercyclical in post-communist countries.

- trade balances (current accounts) are relatively strongly counter-cyclical in post-communist countries.
We understand that business cycle analysis in postcommunist countries is more complex because of differences related to the level of economic (monetary) integration into European structures, their financial links to the rest of the world, etc. However, the results obtained herein reveal that the simple characteristics of business cycles are similar across post-communist countries, with the exception of Romania.

When analyzing business cycles, we attempted to use as long a time series as possible. This analysis focused on the characteristics of the economic cycles of European post-communist countries. Our intention was thus partly thwarted by the lack of sufficiently long and reliable data for these economies. Nevertheless, this paper identifies particularities in these postcommunist countries despite the data limitations. We also wanted to contrast our results with previously known and accepted conclusions regarding other types of economies that are in many ways different from post-communist economies. The reasons for these differences are the focus of ongoing research and continuous discussions, and our goal was not to analyze any changes that occurred after the period of Great Moderation. Rather, our intent was to use the available data on European post-communist countries to reveal the characteristics of their economic cycles. Furthermore, we tried to intuitively form conclusions regarding why a group of European post-communist countries differs from its closest typological group, i.e., small emerging economies.

\section{References}

Aguiar, M., \& Gopinath, G. (2007). Emerging market business cycles: The cycle is the trend. Journal of Political Economy, 115(1), 69-102.

Burns, A. F., \& Mitchell, W. C. (1946). Measuring business cycles. New York, NY: National Bureau of Economic Research. Retrieved from http://papers. nber.org/books/burn46-1

Calderón, C., \& Fuentes, R. (2010). Characterizing the business cycles of emerging economies (Policy Research Working Paper No. 5343). The World Bank. Retrieved from https://papers.ssrn.com/ sol3/papers.cfm?abstract_id $=1629052$

Eurostat (2014). database (2014). GDP and main components (output, expenditure and income). 
Retrieved from http://appsso.eurostat.ec.europa. eu/nui/show.do?dataset=namq_10_gdp\&lang=en

García-Cicco, J., Pancrazi, R., \& Uribe, M. (2010). Real business cycles in emerging countries? American Economic Review, 100(5), 2510-2531.

Hodrick, R. J., \& Prescott, E. C. (1997). Postwar U.S. business cycles: An empirical investigation. Journal of Money, Credit and Banking, 29(1), 1-16.

Kydland, F. E., \& Prescott, E. C. (1982). Time to build and aggregate fluctuations. Econometrica, 50(2), $1345-1370$

Lucas, R. E. Jr. (1976). Econometric policy evaluation: A critique. Carnegie-Rochester Conference Series on Public Policy, 1(1), 19-46.

Lucas, R. E. Jr. (1977). Understanding business cycles. Carnegie-Rochester Conference Series on Public Policy, 5(1), 7-29.

Mendoza, E. (1991). Real business cycles in a small open economy. American Economic Review, 81(4), 797-818.

Montiel, P. J. (2013). Macroeconomics in Emerging Markets ( $2^{\text {nd }}$ ed.). New York, NY: Cambridge University Press.

OECD database (2014). Quarterly National Accounts. Retrieved from http://stats.oecd.org/BrandedView.aspx?oecd_bv_id=na-data-en\&doi=data00017-en

Ravn, M. O., \& Uhlig, H. (2002). On adjusting the Hodrick-Prescott filter for the frequency of observations. The Review of Economics and Statistics, 84(2), 371-375.

Szomolányi, K., Lukáčiková, A., \& Lukáčik, M. (2011). The $\beta$-convergence of the EU27 countries. In M. Dlouhý \& V. Skočdopolová (Eds.), Proceedings of the 29th International Conference Mathematical Methods in Economics 2011 (Vol. 2, pp. 287-291). Praha: Professional Publishing.

Szomolányi, K., Lukáčik, M., \& Lukáčiková, A. (2014a). Slovak Economy and Interest Rates Shocks. In M. Reiff, \& M. Lukáčik (Eds.), Proceedings of the 17th International Conference Quantitative Methods in Economics. Multiple Criteria Decision Making XVII (pp. 263-269). Bratislava: Ekonóm.

Szomolányi, K., Lukáčik, M., \& Lukáčiková, A. (2014b). World Financial Frictions and the Czech Economy. In O. Deev, V. Kajurová, \& J. Krajíček (Eds.), Proceedings of the 11th International Sci- entific Conference European Financial Systems (pp. 638645). Brno: Masaryk University.

Uribe, M., \& Schmitt-Grohé, S. (2017). Open Economy Macroeconomics. Princeton, NJ: Princeton University Press.

Uribe, M., \& Yue, V. Z. (2006). Country spreads and emerging countries: Who drives whom? Journal of International Economics, 69(1), 6-36.

Williamson, S. D. (2014). Macroeconomics ( $5^{\text {th }}$ ed.). Upper Saddle River, NJ: Pearson Education.

\section{Endnotes}

1 Longer data series were not published at the time of processing.

2 Croatia was not a member of the European Union at the time of the paper's completion (2011). Hungary seemed to converge to a statistically significantly lower steady state than other European Union members.

\section{Acknowledgements}

This paper is supported by the Grant Agency of the Slovak Republic - VEGA, grant no. 1/0444/15 “Econometric Analysis of Production Possibilities of the Economy and the Labour Market in Slovakia". 
